$$
\begin{aligned}
Z_{4}{ }^{\prime}(x)=(2 / \pi x)^{\frac{1}{2}} \exp \left(-x / 2^{\frac{1}{2}}\right) & \\
\cdot & \cdot\left\{(-x) \cos \left(x / 2^{\frac{1}{2}}-\pi / 8\right)+V(-x) \sin \left(x / 2^{\frac{1}{2}}-\pi / 8\right)\right\} .
\end{aligned}
$$

The constants $c_{i j}, d_{i j}$, and $e_{i j}(1 \leqq i \leqq t, 0 \leqq j \leqq 8)$ are given in Tables 1 to 3 .

Battersea College of Technology

London, S.W.11.

1. H. H. Lowell, Tables of the Bessel-Kelvin Functions Ber, Bei, Ker, Kei, and their Derivatives for the Argument Range 0(0.01)107.50, Technical Report R-32, National Aeronautics and Space Administration, Washington, D. C., 1959.

2. L. N. Nosova, Tables of Thomson Functions and their First Derivatives, Izdatel'stvo AkademiY Nauk SSSR, Moscow, 1960.

3. National Bureau of Standards Applied Mathematics Series, v. 9, Tables of Chebyshev Polynomials $S_{n}(x)$ and $C_{n}(x)$, U.S. Government Printing Office, Washington, 1952.

\title{
Quadrature Formulas over Infinite Intervals in Terms of Differences
}

\section{By F. D. Burgoyne}

This paper describes quadrature formulas which may be used instead of GaussLaguerre or Gauss-Hermite formulas and in which differences are employed. The relative merits of each type of formula are considered and simple examples are given. While the idea behind them is certainly not new, the formulas themselves do not appear to have been previously published.

An integral of the type $\int_{0}^{\infty} e^{-x} f(x) d x$ if often evaluated numerically by means of a Gauss-Lgguerre quadrature formula. Thus we have

$$
\int_{0}^{\infty} e^{-x} f(x) d x=\sum_{i=0}^{n} H_{n i} f\left(a_{n i}\right)+E,
$$

where the weights $H_{n i}$ and the abscissas $a_{n i}$ have been extensively tabulated (see, for example, [1]), and $E$ represents an error term. Similarly, an integral of the type $\int_{-\infty}^{\infty} e^{-x^{2}} f(x) d x$ can be evaluated numerically by a Gauss-Hermite quadrature formula, and we have

$$
\int_{-\infty}^{\infty} e^{-x^{2}} f(x) d x=\sum_{i=0}^{n} K_{n i} f\left(b_{n i}\right)+F,
$$

where the $K_{n i}$ and the $b_{n i}$ have also been the subject of several tabulations (one of the most comprehensive being [2]), and $F$ is the error term. The advantage of such formulas is that for a given value of $n$ they attain the maximum possible degree of precision, i.e. $2 n+1$, and on this account they are usually very accurate. Further, if an automatic computer is being used, it may be convenient not to have to form differences.

Unfortunately they also suffer from several drawbacks. Perhaps the most serious is that having arrived at an estimate for the value of an integral using a certain value of $n$, if we then decide to use a different value of $n$ we have to repeat the entire calculation with new weights and abscissas. Although this is undesirable

Received September 24, 1962. 
in hand computation, it is even more so if an automatic computer is being used: the program will have to be supplied with several sets of weights and abscissas. From the point of view of the desk machine user another drawback is that both weights and abscissas are usually awkward irrational numbers. Moreover, if $f(x)$ is only known at a set of equidistant abscissas the use of a Gauss-Laguerre or a Causs-Hermite formula will involve us in interpolation to find $f\left(a_{n i}\right)$ or $f\left(b_{n i}\right)$. The quadrature formulas to be described do not suffer from these defects.

Let $w$ be the interval at which $f(x)$ is tabulated. We shall use the following notation for factorials:

$$
\begin{array}{ccc}
x^{(0)}=1, \quad x^{(i)}=x(x-1) \cdots\{x-(i-1)\} & i=1,2, \cdots, \\
x^{\{1\}}=x, \quad x^{\{2 i-1\}}=x\left(x^{2}-1\right) \cdots\left\{x^{2}-(i-1)^{2}\right\} & i=2,3, \cdots, \\
x^{[0]}=1, \quad x^{[2 i]}=x^{2}\left(x^{2}-1\right) \cdots\left\{x^{2}-(i-1)^{2}\right\} & i=1,2, \cdots, \\
x_{(i)}=x^{(i)} / i ! \text { etc. } &
\end{array}
$$

In what follows $f^{\prime}(x)$ will be assumed to be differentiable as many times as is required.

From Newton's Forward Difference interpolation formula we obtain

$$
\int_{0}^{\infty} e^{-x} f(x) d x=\sum_{i=0}^{n} h_{i} \Delta^{i} f(0)+e
$$

where

$$
h_{i}=\int_{0}^{\infty} e^{-x}(x / w)_{(i)} d x=w \int_{0}^{\infty} e^{-w x} x_{(i)} d x
$$

and

$$
e=w^{n+1} \int_{0}^{\infty} e^{-x}(x / w)_{(n+1)} f^{(n+1)}(\xi) d x
$$

$\xi$ being a positive number depending on $n, w, x, f(x)$. We see that (3) represents a quadrature formula to replace (1), in which the abscissas are $0, w, \cdots, n w$. Each term in the summation on the right hand side of (3) represents a correction to the previous estimate and involves us in the use of one new abscissa. It will thus be evident from the magnitude of this correction at what stage we have considered sufficient terms to estimate the value of an integral to a given accuracy. If, as sometimes happens, the resulting series is slowly convergent while the terms alternate in sign, we may conveniently sum the series $b_{y}$ Euler's transformation or a similar device. Whereas the Gauss-Laguerre ordinates $f\left(a_{n i}\right)$ cannot be checked for numerical slips by straight differencing, the formation of the difference table for use with (3) will automatically provide a check that we have evaluated $f(i w)$ correctly. The degree of precision of (3) is plainly $n$.

Perhaps the easiest way to evaluate $h_{i}$ is from the recurrence relation

$$
h_{i}=\frac{1}{w} \sum_{j=1}^{i}(-1)^{j-1} h_{i-j} / j \quad i=1,2, \cdots
$$


starting with $h_{0}=1$. This relation may be derived as follows:

$$
\begin{aligned}
h_{i} & =\frac{w}{i !} \int_{0}^{\infty} e^{-w x} x^{(i)} d x=\frac{1}{i !} \int_{0}^{\infty} e^{-w x}\left\{D x^{(i)}\right\} d x \quad \text { (by partial integration) } \\
& =\frac{1}{i !} \int_{0}^{\infty} e^{-w x}\left\{i x^{(\imath-1)}-i(i-1) x^{(i-2)} / 2+\cdots+(-1)^{i-1} i ! / i\right\} d x \\
& =\frac{1}{w} \sum_{j=1}^{i}(-1)^{j-1} h_{i-j} / j .
\end{aligned}
$$

We may thus tabulate the $h_{i}$ for any given interval $w$, but if an automatic computer is being used it will probably be simpler to generate them in the program.

Using the methods of [3] it is possible to derive from (4) an expression for $e$, the leading term of which is $h_{n+1} w^{n+1} f^{(n+1)}\left(\xi^{\prime}\right), \xi^{\prime}$ being a positive number depending on $n, w, f(x)$. The details are omitted here since the result is of little practical importance, although it does enable a comparison to be made between $e$ and the error term of a different quadrature formula having the same degree of precision. Another fact which emerges is that $e$ is largely independent of $w$, and this is borne out by numerical examples.

To obtain a similar quadrature formula to replace (2) we consider Stirling's interpolation formula, and we find that

$$
\int_{-\infty}^{\infty} e^{-x^{2}} f(x) d x=\sum_{i=0}^{n} k_{i} \delta^{2 i} f(0)+f
$$

where

$$
k_{i}=\int_{-\infty}^{\infty} e^{-x^{2}}(x / w)_{[2 i]} d x=2 w \int_{0}^{\infty} e^{-w^{2} x^{2}} x_{[2 i]} d x
$$

and

$$
f=w^{2 n+2} \int_{-\infty}^{\infty} e^{-x^{2}}(x / w)_{[2 n+2]} f^{(2 n+2)}(\eta) d x=k_{n+1} w^{2 n+2} f^{(2 n+2)}\left(\eta^{\prime}\right)+\cdots,
$$

$\eta, \eta^{\prime}$ being numbers depending on $n, w, x, f(x)$ and $n, w, f(x)$ respectively. Corresponding to $(5)$ we have the relation

$$
k_{i}=\left(k_{i-1}-k_{i-2} / 6+k_{i-3} / 30-\cdots\right) / 4 i w^{2} \quad i=1,2, \cdots,
$$

which, starting with $k_{0}=\pi^{\frac{1}{2}}$, may be used for the evaluation of $k_{i}$. The proof is similar to that of (5). Since the coefficients of $k_{i-j}$ are awkward numbers, to evaluate $k_{i}$ it will probably be easier to express $x_{[2 i]}$ in terms of powers of $x^{2}$ (see [4]) and to use the result that

$$
2 w \int_{-\infty}^{\infty} e^{-w^{2} x^{2}} x^{2 j} d x=\left(j-\frac{1}{2}\right) ! / w^{2 j}=(2 j-1) ! ! \pi^{1 / 2} / 2^{j} w^{2 j} .
$$

If desired, a similar procedure may be used to evaluate $h_{i}$, in which case we use the result that

$$
w \int_{0}^{\infty} e^{-w x} x^{j} d x=j ! / w^{j}
$$


Although the $k_{i}$ are more troublesome to calculate than the $h_{i}$, they will be found, unlike the latter, to diminish quite rapidly.

We shall now consider two simple examples to illustrate the use of (3) and (i) respectively. Firstly, suppose it is required to evaluate

$$
I=\int_{0}^{\infty} \frac{e^{-x}}{100+2 x} d x
$$

to six decimal places, using $w=\frac{1}{2}$. The estimates given by (3) for $n=0,1,2,3$ are respectively $0.01000000,0.00980198,0.00980780,0.00980757$. We thus infer that to the accuracy required, $I=0.009808$ : this is in full agreement with the exact value. If instead of using (3) we had used (1), and followed a similar procedure, three separate calculations would have been required to obtain $I$ to this accuracy, as the estimates given by ( 1 ) for $n=0,1,2$ are respectively $0.00980392,0.00980755$, 0.00980756 . To illustrate the use of $(6)$ consider the problem of evaluating, as accurately as possible, the integral

$$
J=\int_{-\infty}^{\infty} e^{-x^{2}} J_{0}(x) d x
$$

where $J_{0}(x)$ is the Bessel function of the first kind and order zero, given only that $J_{0}(0)=1, \quad J_{0}( \pm 1)=0.765198, J_{0}( \pm 2)=0.223891$. From (6) we obtain $J=1.570389$ with $n=2$. This compares with the true value of $J=1.570301$, to six decimal places, as given in [5]. With the values of $J_{0}(x)$ given here, there is clearly no point in attempting to evaluate $J$ by Gauss-Hermite quadrature.

Battersea College of Technology

London, S.W.11.

1. National Bureau of Standards Applied Mathematics Series, v. 37, Tables of Functions and of Zeros of Functions, Washington, D.C., 1954.

2. H. E. Salzer, R. Zucker \& R. Capuano, "Table of the zeros and weight factors of the first twenty Hermite polynomials," J. Res. Nat. Bu. Standards, v. 48, 1952, p. 111-116.

3. J. F. Steffensen, Interpolation, Chelsea, New York, Second Edition 1950.

4. R. A. Buckingham, Numerical Methods, Pitman, London, 1957, p. 568-573.

5. G. N. Watson, Theory of Bessel Functions, Cambridge University Press, Second Edition, 1944, p. 394 . 